\title{
ON THE USE OF A CONTINUOUS ACCESSIBILITY ASSESSMENT PROCESS FOR DEALING WITH WEBSITE EVOLUTION
}

\author{
Aline Bossi Pereira da Silva, Celmar Guimarães da Silva and Regina Lúcia de Oliveira Moraes \\ School of Technology, University of Campinas, Limeira, São Paulo, Brazil
}

\begin{abstract}
The accessibility of the websites is important to ensure the full access of relevant information to people with disabilities. Website evolution impacts the website accessibility, as the amount of accessibility faults in websites tend to increase during this phase. Normally, the accessibility evaluation is done before deployment but there is a lack of accessibility maintenance after evolution. Although legislation has been established for government websites, updates are not always conducted. This paper hypothesizes that adopting a continuous validation process of website accessibility could facilitate accessibility maintenance after each evolution. For this end, a tool was adapted to support developers on finding and fixing the accessibility faults. We invited governmental websites managers to participate on our evaluation procedure that was based on failures report by email, questionnaire and direct contact, to make them aware of the regulation and the faults found in the websites under their responsibility. Even after our contact and constant messages sent by email suggesting how to fix the faults the adhesion was low. The results suggested a notable lack of interest towards the regulation compliance and a strong lack of sensitivity towards people's disabilities.
\end{abstract}

\section{KEYWORDS}

Accessibility Evaluation, Accessibility Tools, Website Accessibility Assessment

\section{INTRODUCTION}

Providing web accessibility is an important goal that every website should pursue in order to increase the amount of people that can use it effectively. The main beneficiaries of web accessibility are people with vision, hearing or physical disabilities (Cunningham, 2012). A website that provides accessible content allows people with disabilities to assimilate and understand this content, navigate through it, interact with it without technical restrictions, and even contribute to it (Tanaka and Rocha, 2011; Freire et al., 2008b). Applying accessibility rules on websites can make web content available for all users, including people with disability or temporary limitation (Luján-Mora et al., 2014).

Considering the relevance of this issue, some countries created legislations to reinforce and even compel websites to provide full access by people with disabilities. In Brazil, the expansion of the Internet and the dissemination of Brazilian government information and services on the Internet have influenced the decision to create an accessibility regulation, given that this requirement strongly contributes to the digital and social inclusion of citizens (Bach et al., 2009). Indeed, 23.9\% of the Brazilian people has visual, auditory, motor or intellectual (temporary or not) disabilities (Oliveira, 2012). Therefore, there is a significant part of citizens that may not access all information provided by non-accessible websites.

Two Brazilian laws define website accessibility as a mandatory requirement of public administration websites. They were introduced in 2000 (Laws 10,048 and 10,098) and they were regulated in 2004 (Decree 5,296). After more than a decade of these laws and regulation be placed, it is very easy to find non-accessible Brazilian government's websites. Even when they have some accessibility certificate, they do not remain accessible over time.

Currently, there are tools available for testing the adherence of websites to accessibility rules. However, people that are responsible for website maintenance usually run these tests only once, at the end of the development process. Afterwards, constant changes implemented daily by the users tend to insert accessibility faults. After these changes, website maintenance-workers often do not revalidate their websites, neither using 
experts review nor specific tools. This lack of website evaluation process avoids meeting accessibility standards and may cause a regression in website quality.

Aware of this problem, the goals of this work were: (1) to define a continuous accessibility validation process that periodically informs website managers about the compliance of the website with accessibility rules; and (2) to test the hypothesis that adopting this process would motivate those managers to keep websites accessible, despite maintenance and modifications made throughout their existence. We adapted an accessibility tool for doing this kind of validation and executed experiments that helped us to test this hypothesis.

Considering the experiments, we applied tests on two samples. The goal was to identify the faults that are inserted during website maintenances. In addition, an email with a questionnaire was sent to the focused website managers. The results show that feeding the website maintainers with data about accessibility weaknesses tools was not enough for engaging them in a process of accessibility enhancement. We concluded that there is a gap between the enactment of accessibility laws and a real interest of governmental institutions on implementing them.

This paper is organized as follows. In Section 2, the main Web accessibility concepts and related works are presented. The research methodology is explained in Section 3. In Section 4 the experiments' results and discussions are presented. Finally, Section 5 presents the conclusions and future work.

\section{BACKGROUND AND RELATED WORK}

The investigation of the accessibility during the evolution of websites and the study about the low adhesion to the accessibility regulation have been an important target pursued by several research projects.

Santana and Paula (2013) performed accessibility tests considering two samples. The first one is composed by a selection of 1000 websites, which were chosen in a ranking that classifies the websites based on their quality (popular websites), while the second sample is a random selection of the same amount of websites. Considering the random sample, $14.89 \%$ (around 148 websites) are accessible (i.e., compliant with the guidelines); in the other hand, considering the sample with popular websites, only 4.34\% (around 43 websites) are accessible. The authors' conclusion refers to the evolution of websites. The accessibility non-conformance is high in the popular websites because their contents are dynamic and constantly change, causing an increase in accessibility faults. Some works are focused on governmental websites. Ferreira et al. (2007) presented an accessibility study based on the websites of Brazilian public organizations. According to the law, these websites must be compliant with the W3C accessibility rules (WCAG) and the Brazilian recommendations (e-MAG). The work evaluates the degree of adherence of these websites to the accessibility guidelines. The authors realized that the number of websites that comply with these guidelines decreased, which can be due to the difficulty of keeping websites accessible when they are constantly updated.

Other countries have low rates of accessibility in governmental websites as well. Shah and Shakya (2007) presented a work that evaluated $27 \mathrm{Nepal} \mathrm{governmental} \mathrm{websites.} \mathrm{They} \mathrm{observed} \mathrm{a} \mathrm{very} \mathrm{low} \mathrm{compliance} \mathrm{with}$ the recommendations as only $11.1 \%$ of the websites comply with the regulations. The authors concluded that Nepal, in 2006, needed to make an effort to improve the website accessibility.

Luján-Mora et al. (2014) also addressed the lack of accessibility on governmental websites. They indicate that these sites are not always ready to provide services to people with disabilities. These authors evaluated the accessibility of a group of e-government websites from all South America countries and Spain. Based on the obtained results, they observed that all evaluated websites presented some non-compliance with accessibility laws.

Al-Soud and Nakata (2010) evaluated 30 Jordanian governmental websites in terms of accessibility, usability, transparency and agility. The results show low adherence to the accessibility rules in websites and concluded that those involved in the project are not sufficiently aware of the accessibility guidelines (i.e. there is a lack of training).

Freire et al. (2008a) conducted an exploratory research through the application of a questionnaire that involved the 27 Brazilian states. In the project's development, the authors suggested the lack of professional training and information by those responsible for websites development and test. This is the same suggestion presented by Santana and Paula (2013), which also concluded this lack of professional training and observed that between 85 up to $95 \%$ of the focused websites present any type of accessibility faults. 
There are different ways to evaluate the accessibility of a website. $\mathrm{Vu}$ et al. (2012) highlighted the differences between automatic, semiautomatic and manual evaluation. The automatic evaluation considers client-side and display the non-conformity and suggestions to fix the faults. Semiautomatic tools require a personal intervention and accessibility knowledge as it is necessary to interpret the textual recommendation and to identify the items not included in the tool report.

Website accessibility monitoring can be done in different ways, either in real time, regular intervals, or specific stages of the website development. The work of Fong and Meng (2009) stated that governmental websites represent not only a public image but also a reliable service platform for many users. The authors presented the best practices, models, tools and techniques for measuring, monitoring, and maximizing the efficiency and effectiveness of e-Government services. They also created a real time web-based system to monitor the performance and status of public services, given that they consider monitoring as an essential phase of a web system lifecycle.

There are several tools to validate the website accessibility. DaSilva (Acessibilidade Brasil, 2017) was the first tool available to analyze accessibility of the Portuguese language websites. The recommendations considered by the tool are based on the principles of WCAG 1.0 (W3C, 1999) and 2.0 (W3C, 2008) and the Brazilian recommendations (e-MAG), allowing the analysis of all the pages of the website and indicating the non-conformity with the recommendations. The validator VaMoLà (VaMoLà Project, 2010), automatically verifies some accessibility requirements based on the recommendations of WCAG 2.0 and the requirements of Italian laws. Tanaguru (Tanaguru Project, 2014) is a website accessibility assessment tool that tests the recommendations of WCAG 2.0, Section 508 (United States) and AccessiWeb.

The AChecker tool (Inclusive Design Institute, 2017) checks individual HTML pages for compliance to accessibility standards and we elected it to be adapted to support our proposal. The tool supports the international recommendations WCAG 1.0 and 2.0, and regional recommendations such as BITV 1.0 (Germany), Section 508 (United States) and Stanca Act (Italy).

It is worth noting that this review does not found a work that defined a continuous evaluation process, as well as no tool that makes continuous evaluation of accessibility automatically to motivate website managers to ensure the accessibility of their websites. The next section presents the research methodology followed in this work.

\section{RESEARCH METHODOLOGY}

This section presents the research methodology used for the development of this work. First, we present how we chose the tool and how the samples used in the experiments were composed.

\subsection{The Tool Selection}

The main characteristic of the tool to be selected is the license type, which must be open source. For the development of this work, it was imperative that the tool can be developed in a collaborative public manner, the source can be available for studies, and modifications and redistribution of the software to anyone and for any purpose be allowed. We considered only open source tools in this work as an imperative requirement. This choice enables a collaborative development of the tool, and the availability of its source code for studies, modifications and redistribution.

Considering the tools presented in Section 2, both VaMoLà and AChecker fit the project requirements. VaMoLà considers the WCAG 2.0 recommendations and it is also customized to Italian law. The AChecker tool is able to test the universal recommendations (WCAG 1.0 and WCAG 2.0), enabling more global information testing report. Additionally, the AChecker tool suggests possible corrections to fix the accessibility faults found. This feature can be considered a support for the training process, which can minimize the weaknesses highlighted by some authors (Al-Khalifa and Al-Khalifa, 2011 and Bailey and Pearson, 2010). Therefore, the AChecker tool was selected to be adapted to fulfill the requirements of this work. 


\subsection{The Samples Selection}

As already discussed, the goal of this work was to test whether adopting a continuous accessibility validation process over time (i.e. considering evolution of websites) would help to maintain a website accessible. With that goal in mind, we defined that the first sample is composed by governmental websites that had already undergone accessibility tests. We have chosen these websites because it is assumed that they need to attend the Brazilian accessibility laws (under the decree $n^{\circ}$. 5,296). The information about the previous evaluation was extracted from the Clareou website (Santos and Bastos, 2017), which classifies the websites based on their accessibility level. Therefore, 21 websites from the government category considered accessible by the Clareou website were select as this first sample.

The second sample has all of the City Halls' websites of the State of São Paulo. We submitted these websites to a previous evaluation with the support of the AChecker tool, which we adapted in the context of this work. The process of adapting AChecker to make it a continuous evaluation tool involves changing the database structure and the source code of the tool. We developed and added specific classes and methods to meet research needs. For the automation of accessibility tests, a cron job has been configured on the server to run every day. The new classes are responsible for database connection, and for the management of data about the monitored websites and the frequency of monitoring activities.

The first evaluation aimed to record the existent accessibility faults to compare to the evaluation results over time. The initial sample has 645 websites that were submitted to accessibility tests during 45 days in the period of December 2016 through March 2017.

\subsection{Experiments using the First Sample}

Although Clareou classified the 21 websites used in the first sample as free of accessibility faults, they were submitted to a new test supported by the adapted AChecker tool. The goal of this preliminary test is to reinforce the websites quality under the analysis of the adapted AChecker tool. Thus, the aim is to avoid discrepancy between the implementation of both tools (Clareou and AChecker) which could impact the results. In addition, there was a lack of guarantee that the classification provided by Clareou tool refers to the last version of the focused websites. This procedure showed to be appropriate since there were seven websites unavailable (some files were not found and errors were displayed) and only two websites did not show accessibility faults.

After capturing data from the remaining websites (12 websites), half of them (Group 1) was randomly selected to receive weekly emails informing the research context, the accessibility failures found and suggesting how to fix the corresponding faults.

The reason for choosing half of the sample was to create a control group of websites (Group 2). It would help to verify if the awareness about the accessibility faults would influence the accessibility improvement, allowing a comparison between the aware and the unaware about the accessibility faults.

During 30 weeks, the experiments were performed checking 6 websites and registering the number of accessibility failures found in each week to each website for further analysis.

\subsection{Experiments using the Second Sample}

We evaluated preliminarily the second sample (City Halls' websites) for 45 days. During these preliminary tests, the number of accessibility failures were recorded for each website in order to compare with the data captured during the tests period. During the test the adapted AChecker sent email to the websites managers to make them aware about the accessibility failures found.

After this period, the websites were grouped considering the size-based distribution of cities according to the last census of the IBGE (Instituto Brasileiro de Geografia e Estatística - The Brazilian Institute of Geography and Statistics). We hypothesize that large cities could have more resources available than smaller ones, and that this fact could influence how much effort they could put on accessibility issues. The census classifies $88.3 \%$ of São Paulo cities as small cities, $10.3 \%$ as medium cities and $1.4 \%$ as large and metropolis cities (IBGE, 2010). First, the 645 websites were divided in two groups: the test group and the control group. The test group was divided in other 3 groups. So, each of the tested groups was composed by 99 websites 
randomly selected, preserving the distribution previously mentioned (i.e. 88 small cities, 10 medium cities and 1 large city). The groups have no intersection, i.e., each website belongs to only one group.

On different dates, each group was set up to receive emails reporting the research context, the accessibility failures found by AChecker tool and the suggestion to fix the corresponding faults. The emails were sent weekly during 30 weeks. At the end of this period, all managers of the tested websites received a questionnaire. It highlighted the importance of accessibility and the main goal of the research (i.e., to investigate whether governmental websites take into consideration the WCAG 2.0 recommendations) and invited them to answer a set of questions related to the awareness of this context and of the emails that were sent.

The questionnaire involved multiple choice and open-ended questions. The questions focused on verifying whether the participant was aware of the Brazilian laws that establish a deadline for the accessibility of all public administration sites. In addition, some questions tried to identify which are the difficulties for the adequacy of the websites.

In addition to the questionnaire, as a complementary evaluation, $2.5 \%$ of the tested websites of the second sample and $2.5 \%$ of untested websites were randomly selected to receive a direct contact (via phone) to understand the accessibility training level, as well as to invite them to participate in the research by answering the questionnaire. The next section presents the results and discussions about the experiments.

\section{RESULTS AND DISCUSSION}

In the first sample, after testing websites from Group 1 (6 governmental websites) with the adapted AChecker, the results obtained were compared with the results of the training period (i.e. the period before the emails were sent to the website managers). It was observed that there was a decrease in the number of failures in $34 \%$ of the websites tested, no changes in $16 \%$ of them, and an increase in $50 \%$ of them. Observing the results in more detail, only one website presented a significant decrease of $65.74 \%$ in the accessibility failures. The highest increase was $17.17 \%$ in another website. The websites of Group 2, also in the first sample, continued to be tested but no email was sent to the managers. Considering the same period (training and 30-weeks experiment period) it was observed that in $60 \%$ of the websites the accessibility failures decreased while there was an increase in $40 \%$ of them, and no website $(0 \%)$ maintained the same number of failures. It is important to highlight that although the percentage of websites with decrease in the number of failures is significantly higher than the websites of Group 1, only one website presented an expressive decrease while the others had a very small decrease. Table 1 presents the results of each group.

Table 1. Comparative failure statistics of first sample

Table 2. Comparative failure statistics of second sample

\begin{tabular}{|c|c|c|}
\hline & Group 1 & Group 2 \\
\hline Decreased & $34 \%$ & $60 \%$ \\
\hline Increased & $16 \%$ & $0 \%$ \\
\hline Remained & $50 \%$ & $40 \%$ \\
\hline
\end{tabular}

\begin{tabular}{|c|c|c|c|c|}
\hline & Group 1 & Group 2 & Group 3 & Group 4 \\
\hline Decreased & $44 \%$ & $41 \%$ & $47 \%$ & $48 \%$ \\
\hline Increased & $38 \%$ & $40 \%$ & $43 \%$ & $39 \%$ \\
\hline Remained & $18 \%$ & $19 \%$ & $10 \%$ & $13 \%$ \\
\hline
\end{tabular}

In the second sample, as discussed in the previous section, the websites were separated in four groups, and three of them were submitted to accessibility tests. After capturing initial data (training period), the adapted AChecker started to send a weekly email to the website managers. The email reported the accessibility failures found, their severity and suggestions for corrections. Table 2 presents the results of each group. Note that the fourth group is the group of 321 websites that did not receive emails. It is possible to observe that the results are very similar, with a failure decrease percentage varying from 41 up to $48 \%$, a failure increase percentage from 38 up to $43 \%$, and 10 up to $19 \%$ remained stable (the same amount of failures). Based on these results it was not possible to identify a significant difference among the groups, even considering the group that received no email. It is worth noting that a stable number of errors does not mean that they are the same faults (it was not checked the faults per se); a more complete checking could check if the same failures remained or not.

After the experiment periods of each group, a questionnaire was sent by email to 297 website managers twice a week during 9 weeks. Amongst those, $2.5 \%$ of the websites that received the failures notification and $2.5 \%$ of the websites that received no emails were randomly chosen and their managers were directly contacted 
(by phone). The purpose of this contact was to raise the awareness of the website managers related to the research and to the accessibility issues. The results obtained in this stage were the following:

- $10 \%$ of the emails sent with the questionnaire attached were opened (30 emails)

- $1 \%$ of the emails sent were triggered ( 3 emails)

- $12.79 \%$ of the emails received requested removal of the mailing list (38 emails)

- $5.05 \%$ of the emails received answered the questionnaires (15 emails)

- $1.34 \%$ of the emails were answered after direct contact (phone) (4 emails).

The websites that received emails and the phone calls ( 8 websites) presented the following results before the calls:

- $75 \%$ are interested but asked to forward the email to someone else (6 websites)

- $13 \%$ have no interest in the research (1 website)

- $13 \%$ had already received the questionnaire and reports (1 website)

The same websites after the phone calls, when the websites manager were re-invited to answer the questionnaire, the same websites returned the following

- $25 \%$ answered the questionnaire after the call (2 websites)

- $75 \%$ did not respond to the questionnaire even after the call (6 websites)

Other eight websites that were untested (i.e. received no email) when directly contacted returned by phone the following results:

- $63 \%$ are interested in receiving the notifications and the questionnaire (5 websites)

- $\quad 38 \%$ have no interest in the research ( 3 websites).

The emails with the questionnaire in attachment were sent to the website managers after the call and the results obtained are as follows:

- $25 \%$ answered the questionnaire after the call (2 websites)

- $75 \%$ did not respond to the questionnaire even after the call (6 websites).

According to the received answers (15 answers) of the applied questionnaire, the results indicate that $66.7 \%$ of the website managers are aware about the law and tried to apply it, only $26.7 \%$ have no knowledge about the laws and $6.6 \%$ are aware about the law but are unable to apply it. Considering the participants, $60 \%$ already regularly received the accessibility reports generated by the AChecker tool.

Regarding the website improvements during the evaluated period (December 2016 to March 2017), 33\% of respondents were unable to justify accessibility improvements made on their website; $26.7 \%$ said that the website improvements are due to other institution demands; $13 \%$ answered that no improvements were done; $20 \%$ stated that the website improvements were done to attend the institution demands and the faults reported by AChecker tool as well; and only $6.7 \%$ reported that the improvements were made considering only the report of failures sent by AChecker tool.

When the managers were questioned about the reason why the websites remained with accessibility faults, $40 \%$ of them were not able to identify the reason, $20 \%$ reported that there were other priorities in the organization, $13.3 \%$ answered that they did not receive the accessibility reports that were sent during the experiment period, $6.7 \%$ reported that the website was being updated, $6.7 \%$ admitted that the website needs to be completely redone and this task will be completed soon, and only $13.4 \%$ answered that they fixed the faults responsible by the failures.

Regarding the usefulness of receiving the reports to make accessibility improvements, $80 \%$ of the participants thought it was useful to receive them and $20 \%$ of them did not know how to answer this question. Moreover, $86.7 \%$ of the respondents said that they would like to continue receiving the accessibility reports.

When questioned about the difficulty in adapting accessibility rules, $38.5 \%$ of respondents indicated that there are technical difficulties in implementing accessibility improvements, followed by $30.8 \%$, which reported that it was difficult to maintain recommendations during website updates. WCAG accessibility recommendations are difficult to understand to $15.4 \%$ of the respondents, $7.7 \%$ complained about financial problems and $7.6 \%$ indicated lack of knowledge about the recommendations. 


\section{CONCLUSION}

The web is characterized as an important way of communication that promotes the movement of information quickly without people having to move physically. But the lack of accessibility on websites makes it difficult for people with disabilities to navigate, interact with or contribute to it. Accessibility barriers can appear on websites in all stages of their development and especially after their publication. Indeed, daily changes and the insertion of contents by the users tend to place accessibility faults in the website that, if not revalidated (e.g. with the support of accessibility tools), will make the website no longer compliant with the accessibility standards.

The evaluation of the governmental websites was carried out in two samples. The first consisted of 21 websites in the government category that were reported by the Clareou tool website as compliant with the accessibility guidelines. The second sample considered 645 City Halls' websites of the state of São Paulo cities, in Brazil, whose selection was based on IBGE Cidades (IBGE, 2010).

Analyzing the results of the evaluation enabled us to conclude that public Brazilian institutions still have a lack of interest in accessibility. This happens even after more than ten years of the regulation of Decree $n^{\circ}$. 5,296 in December 2004, which established a period of 12 months for public administration websites (public interest or government-funded institutions websites) to comply with those recommendations. On the first and second samples, the evaluated websites had low adherence to accessibility standards. When website managers were notified of the problem by email describing the failures and solutions to fix the faults, we observed no interest by the majority of the managers, even after 7 months of monitoring. Unfortunately, we concluded that the effort to report the failures and guide the managers to fix the faults did not work, as the behavior of the notified websites manager and the ones that were not notified were quite similar.

Considering the institutions that were inquired by phone about the research, only $6.7 \%$ indicated that the improvements were made as a result of the indications in the report sent by the system. Another $20 \%$ reported that there was a lack of priority for these improvements over other tasks. The others considered helpful to receive the reports or advised that they wanted to continue receiving the accessibility reports. However, they did not put any effort to fix the faults.

In addition, it is concluded that there was difficulty in reaching the responsible for the information of the institution on the Internet. In particular, the group of institutions that had direct contacts (phone calls) could not even identify the person responsible for answering the evaluation questionnaire. Thus, the results obtained in this research revealed strong evidence that the hypothesis is false, because even with the continuity of accessibility assessments in websites already published, the faults were still present in the websites of public institutions.

Based on the results, it is possible to assume that the low adherence to the research was due to the lack of priority in the application of Decree no. 5296 that requires that the websites must be accessible. Besides, if an employee participates in the survey and informs that accessibility is not enforced as demanded in regulated laws, he would be admitting to be negligent in their functional responsibility. There was a noticeable lack of interest in the issue of accessibility on websites, even though this is a legal requirement on governmental websites.

Other websites that are not compelled to follow accessibility recommendations could potentially have an even worse accessibility. So, as future work we suggested tests in non-governmental websites in order to identify the knowledge and interest of other areas in relation to the accessibility. In addition, we suggest including the Brazilian recommendations (e-MAG) in the tool for a more comprehensive evaluation.

\section{ACKNOWLEDGEMENT}

We thank the grant \#2019/04222-4 from São Paulo Research Foundation (FAPESP) and the grant \#2232/19 from FAEPEX/PRP/University of Campinas. 


\section{REFERENCES}

Acessibilidade Brasil, 2017. DaSilva - Avaliador de Acessibilidade para Websites. [online] Available at: https://www.dasilva.org.br [Accessed 10 Sep 2017].

Al-Soud, A. R. and Nakata, K., 2010. Evaluating e-government websites in Jordan: Accessibility, usability, transparency and responsiveness. Proceedings of the 2010 IEEE International Conference on Progress in Informatics and Computing. Shanghai, China, pp. 761-765.

Al-Khalifa, A. S. and Al-Khalifa, H. S., 2011. An educational tool for generating inaccessible page examples based on WCAG 2.0 failures. Proceedings of the 2011 International Cross-Disciplinary Conference on Web Accessibility, Hyderabad, India, Article No. 30.

Bach, C. F. et al., 2009. Diretrizes de acessibilidade: uma abordagem comparativa entre WCAG e e-MAG. In Revista Eletrônica de Sistemas de Informação, v. 8, n. 1.

Bailey, C. and Pearson, E., 2010. An educational tool to support the accessibility evaluation process. Proceedings of the 2010 International Cross-Disciplinary Conference on Web Accessibility (W4A). Raleigh, USA, Article No. 12.

Cunningham, K. 2012. Accessibility Handbook: Making 508 Compliant Websites. O'Reilly Media, Inc.

Freire, A. P. et al., 2008. A survey on the accessibility awareness of people involved in web development projects in Brazil. Proceedings of the 2008 International Cross-disciplinary Conference on Web Accessibility (W4A), Beijing, China, pp. 87-96.

Freire, A. P. et al., 2008. Proceedings of the 2008 ACM Symposium on Applied Computing. Fortaleza, Brazil, pp. 2421-2425.

Ferreira, S. B. L. et al., 2007. E-acessibilidade: tornando visível o invisível. In Revista Morpheus-Estudos Interdisciplinares em Memória Social, 6. Article No. 10.

Fong, S. and Meng, H. S., 2009. A web-based performance monitoring system for e-government services. Proceedings of the $3^{\text {rd }}$ International Conference on Theory and Practice of Electronic Governance. Bogota, Colombia, pp. 74-82.

Inclusive Design Institute, 2017. AChecker. [online] Available at: https://achecker.ca/. [Accessed 29 May 2019].

IBGE (Instituto Brasileiro de Geografia e Estatística). Censo 2010, 2010. [online] Available at: http://www.censo2010.ibge.gov.br [Accessed 29 May 2019].

Luján-Mora et al., 2014. Egovernment and web accessibility in South America. Proceedings of 2014 International Conference on eDemocracy \& eGovernment (ICEDEG). Quito, Ecuador, pp. 77-82.

Oliveira, L. M. B., 2012. Cartilha do Censo 2010 - Pessoas com deficiência. Brasília: Sdh-pr/snpd, 17. [online] Available at:http://www.pessoacomdeficiencia.gov.br/app/sites/default/files/publicacoes/cartilha-censo-2010-pessoas-comdeficienciareduzido.pdf [Accessed 29 May 2019].

Santos, R. and Bastos, G., 2017. Clareou. [online] Available at: https://clareou.com/. [Accessed 29 May 2019].

Santana, V. F. and Paula, R. A., 2013. Web accessibility snapshot: an effort to reveal coding guidelines conformance. Proceedings of the 10th International Cross-Disciplinary Conference on Web Accessibility, pp. 2. ACM.

Shah, B. P. and Shakya, S., 2007. Evaluating the web accessibility of websites of the central government of Nepal. Proceedings of the $1^{\text {st }}$ International Conference on Theory and Practice of Electronic Governance. pp. 447-448. ACM.

Tanaguru Project, 2017. Tanaguru Monitor. [online] Available at: http://www.tanaguru.com/en/. [Accessed 29 May 2019].

Tanaka, E. H., and Rocha, H. V., 2011. Evaluation of web accessibility tools. Proceedings of the $10^{\text {th }}$ Brazilian Symposium on Human Factors in Computing Systems and the $5^{\text {th }}$ Latin American Conference on Human-Computer Interaction. Porto de Galinhas, Brazil, pp. 272-279.

VaMoLà Project, 2010. VaMoLà Monitor. [online] Available at: http://sourceforge.net/projects/vamola-monitor. [Accessed 29 May 2019].

Vu, T.-H.-G. et al., 2012. Checking and correcting the source code of web pages for accessibility. Proceedings of 2012 IEEE RIVF International Conference on Computing \& Communication Technologies, Research, Innovation, and Vision for the Future. Ho Chi Minh City, Vietnam, pp. 1-4.

W3C (World Wide Web Consortium), 1999. Web Content Accessibility Guidelines (WCAG) 1.0. [online] Available at: http://www.w3.org/TR/WCAG10. [Accessed 29 May 2019].

W3C (World Wide Web Consortium), 2008. Web Content Accessibility Guidelines (WCAG) 2.0. [online] Available at: http://www.w3.org//TR/WCAG20. [Accessed 29 May 2019]. 